\title{
Metodologias ativas para o ensino da computação: uma revisão sistemática e um estudo prático
}

\author{
Amanda Körber Hartwig, Marlei Maria Silveira, Leonardo Fronza, \\ Mauro M. Mattos, Luciana P. de Araújo Kohler \\ ${ }^{1}$ Laboratório de Desenvolvimento e Transferência de Tecnologia (LDTT) \\ Departamento de Sistemas e Computação \\ Universidade Regional de Blumenal (FURB) - Blumenau, SC - Brazil
}

\begin{abstract}
The growing digital culture in people's daily lives has been influencing the way individuals learn, consult information, participate in groups and exchange experiences with each other. With it, the way the student interacts in the classroom is also changing as he has the information in digital media. From this question, some authors bring experiences, reports and studies in the active methodologies area applied to teaching so that the role between teacher and student is modified. So that, this paper presents a Systematic Literature Review that identified studies in the active learn methologies area and relate theirs with Computing area, relating some examples that how put this methodologies in practice.
\end{abstract}

Resumo. A cultura digital crescente na vida cotidiana das pessoas vem influenciando a forma com que os indivíduos aprendem, consultam informações, participam de grupos e trocam experiências entre si. Com ela, a forma com a qual o aluno interage em sala de aula também está mudando, visto que ele tem as informações nos meios digitais. A partir dessa questão, alguns autores trazem experiências, relatos e estudos na área de metodologias ativas aplicadas ao ensino de modo que o papel entre professor e aluno seja modificado. Dessa forma, este artigo apresenta uma Revisão Sistemática da Literatura que identificou trabalhos na área de metodologias ativas e em seguida, os relaciona a área da Computação, abordando alguns exemplos de como coloca-las em prática.

\section{Introdução}

Metodologias ativas são estratégias de ensino centradas na participação efetiva dos estudantes na construção do processo de aprendizagem, de forma flexível, interligada e híbrida [Bacich and Moran 2018], realizando o aprendizado em três níveis, sendo eles o individual, em grupo e com tutoria. Nessa metodologia, o aluno passa a ser o protagonista do aprendizado, sendo necessário também seu interesse e disposição para a prática do ensino-aprendizado. Dessa forma, com o aprendizado de forma individualizada, é possível promover a personalização do ensino. Essa personalização leva em consideração o tempo de aprendizado de cada aluno, assim como respeita as limitações e talentos de cada um. Além disso, um diferencial que se traz na personalização do ensino, é trazer as questões cotidianas para a sala de aula, de modo que os alunos tenham o objetivo do aprendizado mais próximo de sua realidade.

Para realizar essa personalização de forma adequada e aplicar as metodologias ativas de modo que o aluno seja o protagonista do aprendizado, existem várias estratégias 
VIII Congresso Brasileiro de Informática na Educação (CBIE 2019)

Anais do XXV Workshop de Informática na Escola (WIE 2019)

de ensino, sendo algumas delas sala de aula invertida, rotação por estações, aprendizagem baseada em projetos, aprendizagem baseada em problemas, entre outras. Ainda, junto a essa personalização, é comum que seja abordada a prática do ensino híbrido, isto é, a junção entre tecnologias digitais e de comunicação (TICs) e as atividades presenciais [Bacich and Moran 2018, Virginia 2016].

As metodologias ativas e o ensino híbrido estão ganhando espaço nas salas de aula que buscam a inovação. A partir desses conhecimentos, o professor consegue personalizar o ensino para o aluno de modo que ele aprenda os seus interesses, além de desenvolver melhor suas habilidades em evidência. Nesse contexto, o objetivo geral deste trabalho é identificar quais são as metodologias ativas mais apropriadas para a aplicação no ensino da computação e informática nos cursos de graduação. Já os objetivos específicos são: (a) relacionar os diferentes tipos de metodologias ativas; $(b)$ testar em ambiente de sala de aula as metodologias ativas afim de validar a escolha.

\section{Revisão Sistemática da Literatura}

Para auxiliar na identificação das metodologias ativas apropriadas para a aplicação no ensino da computação nos cursos de graduação, bem como relaciona-las, foi realizada uma revisão sistemática da literatura (Systematic Literature Review - SLR). Para isso, seguiuse o modelo de SLR relatado em [Santos et al. 2012] que divide a SLR nas seguintes etapas: definição de uma Questão Principal (QP), definição de Questões Específicas (QE), definição de uma String de busca, leitura de título, leitura de resumo, leitura diagonal e leitura completa. Para cada etapa de leitura, deve-se tentar responder as QEs e QP, verificando critérios de inclusão e de exclusão para esses artigos.

A etapa 1 da SLR foi executada a partir da leitura do título dos artigos encontrados, excluindo aqueles que não condiziam em responder as QPs e as QEs. Além disso, excluiuse artigos repetidos, artigos pagos e artigos que não estavam disponíveis para download. Após essa primeira etapa, encontrou-se um total de 200 artigos dos 618 iniciais. Após a seleção pelo título, deu-se início a etapa 2 que é a leitura do resumo, com o objetivo de refinar ainda mais a busca. Após a segunda etapa, obteve-se um total de 95 artigos. Por fim, na terceira etapa, cada um dos artigos foi aberto e realizado uma leitura diagonal, ou seja, analisado os títulos internos das seções, com foco na introdução e nos resultados, obtendo-se um total de 66 artigos após essa leitura.

Após a seleção dos 66 artigos, realizou-se a leitura completa desses, com o objetivo de responder as QPs e QEs, sendo que se encontrou apenas 11 artigos ao todo. Os artigos encontrados são os artigos dos autores: [Drini 2018], [Chen and Weng 2008], [Jonsson 2015], [Coldwell et al. 2011], [Abdullah and Yih 2014], [Benlloch-Dualde et al. 2010], [Gouvea et al. 2015], [Krugel and Hubwieser 2017], [Gudigantala 2013] e [Mok 2014].

A partir desses artigos, respondeu-se as questões de pesquisa levantadas, iniciando-se pelas questões específicas. Sendo a "[QE1] Qual o curso que é aplicado?", obteve-se a partir da leitura desses artigos cursos na área da computação, como se esperado, variando entre Sistemas de Informação, Ciência da Computação, Engenharia da Computação, Tecnologia da Informação e Engenharias no geral. Ainda, alguns desses artigos citou a disciplina na qual a metodologia ativa foi aplicada, sendo disciplinas de programação, algoritmos e estrutura de dados, design, redes e sistemas operacionais. A 
VIII Congresso Brasileiro de Informática na Educação (CBIE 2019)

Anais do XXV Workshop de Informática na Escola (WIE 2019)

segunda questão a ser respondida foi "[QE2] Se o curso é presencial ou a distância? (Modalidade de ensino)". Dos onze artigos, apenas um deles se tratava de modalidade a distância. Com relação a terceira pergunta "[QE3] Quais plataformas híbridas são utilizadas?" os que relataram mencionaram ter utilizado Massive Open Online Courses (MOOCs), Google Docs, eLive, Facebook e PodCast. Alguns artigos relataram utilizar hardwares diferenciados como tablets, notebooks, caneta digital e LAN. Ainda, em relação a ferramentas gerais, mencionou-se o uso de quiz, avaliação entre pares, RPGs online, simuladores, galerias de fotos online, wikis, questionários e exercícios integrados em plataformas web, vídeos de forma geral e sistemas colaborativos.

Após responder as perguntas específicas, pode-se responder as perguntas principais, sendo a primeira "[QP1] Quais são e como são utilizadas as metodologias ativas para o ensino da computação nos cursos de tecnologias?”. Com relação as metodologias, os artigos relataram o uso de sala de aula invertida, programação/discussão em pares, aprendizagem apoiada por tablets/notebooks, aprendizagem online, contrato de aprendizagem professor/aluno, feedback instantâneo, aprendizagem baseada em projetos e em problemas e aprendizagem just-in-time. Com relação a aplicação, os artigos expuseram a forma com a qual cada metodologia foi abordada em sala de aula, sendo o principal objetivo motivar os alunos da graduação na disciplina, pois a forma tradicional não os motiva. Além disso, outro ponto colocado é a grande quantidade de reprovações na disciplina com o uso das metodologias tradicionais, o que mudou após a aplicação da metodologia ativa.

A segunda pergunta principal "[QP2] O que falta ser estudado ou aplicado de metodologias ativas na computação?" pode-se responder a partir de alguns dos artigos, porém, nem todos deixaram clara essa informação. Uma informação retirada a partir da leitura dos artigos é que devem existir plataformas melhores para a realização de exercícios de forma interativa. Outra questão apontada foi que deveria-se explorar a respeito de como as tecnologias apropriadas podem ser incorporadas em ambientes de aprendizagem on-line para um ensino eficaz.

\section{Aplicações das metodologias em sala de aula}

Como um dos objetivos da pesquisa é aplicar a metodologia em sala de aula, além dessa SLR foi utilizado o livro de [Bacich and Moran 2018] para o entendimento mais aprofundado a respeito de metodologias ativas. Ainda, baseou-se em [Virginia 2016] e nos artigos de [de Oliveira Pinto 2017] e [de Oliveira Pinto 2019] que definem metodologia ativas e híbridas no ensino básico.

A partir da atualização bibliográfica definiu-se as seguintes metodologias de ensino a serem estudadas para o ensino da computação, sendo: sala de aula invertida (flipped classroom), através do acesso aos conteúdos on-line; rotação por estações; aprendizagem baseada em projetos (Project Based Learning (PBL)); aprendizagem baseada em problemas; estudo de caso e aprendizagem entre pares ou times (Team Based Learning (TBL)).

Foram feitas diversas aplicações dessas metodologias, sendo que na disciplina de Linguagens de Programação do curso de Ciência da Computação trabalhou-se com o PBL e a sala de aula invertida; na disciplina de Interação Humano Computador do curso de Sistemas de Informação aplicou-se um pouco de cada metodologia, afim de varialas; e, da disciplina de Programação III dos referidos cursos aplicou-se a metodologia de aprendizagem baseada em problemas. 
VIII Congresso Brasileiro de Informática na Educação (CBIE 2019)

Anais do XXV Workshop de Informática na Escola (WIE 2019)

Com a aplicação dessas metodologias, pode-se perceber uma maior participação dos alunos em sala de aula, bem como maior engajamento em relação as atividades avaliativas. Com relação a metodologia de sala de aula invertida, pode-se destacar que não foi bem sucedida, pois os alunos não liam o material disponibilizado fora de seu horário de aula. Imagina-se que essa cultura da sala de aula invertida deva ser empregada já em ensino fundamental ou médio, para que permaneça no ensino superior. Com relação as demais práticas, todas foram bem vistas pelos participantes.

\section{Resultados e Discussões}

O trabalho apresentou uma pesquisa com o objetivo de identificar quais são as metodologias ativas mais apropriadas para a aplicação no ensino da computação e informática nos cursos de graduação, relacionando os diferentes tipos de metodologias ativas. Após a revisão sistemática da literatura realizada, observou-se a aplicação das seguintes metodologias para esse contexto: aprendizagem baseada em projetos, aprendizagem baseada em problemas, rotação por estações, programação em pares, sala de aula invertida e aprendizagem colaborativa.

Dessas, as abordagens de aprendizagem baseada em projetos, aprendizagem baseada em problemas, rotação por estações e sala de aula invertida foram aplicadas em diferentes turmas dos cursos de graduação para auxiliar na conclusão do segundo objetivo específico. Desse modo, pode-se destacar que após a aplicação das metodologias, com exceção da sala de aula invertida para o contexto abordado, as demais metodologias são aplicadas ao contexto da computação para o ensino de técnicas e linguagens de programação.

Com relação aos objetivos levantados, o objetivo específico de (a) relacionar os diferentes tipos de metodologias ativas foi alcançado a partir da revisão sistemática da literatura realizada, concluindo-se pela leitura dos onze artigos. Desses, identificou-se que os tipos de metodologias ativas utilizadas para o ensino da computação são: sala de aula invertida, programação/discussão em pares, aprendizagem apoiada por tablets/notebooks, aprendizagem online, contrato de aprendizagem professor/aluno, feedback instantâneo, aprendizagem baseada em projetos e em problemas e aprendizagem just-in-time.

Com relação ao segundo objetivo específico de $(b)$ testar em ambiente de sala de aula as metodologias ativas afim de validar a escolha, algumas das metodologias foram testadas, como relatado no artigo obtendo sucesso em suas aplicações. Contudo, em relação a sala de aula invertida, percebeu-se que alguns dos alunos não se dedicaram nos horários extras a aula, sendo prejudicados com relação a essa metodologia.

A partir da análise dos objetivos específicos pode-se concluir tanto pela leitura dos artigos durante a RSL quanto pela aplicação em sala de aula, que os métodos mais eficientes com melhores resultados são a aprendizagem baseada em problemas e em projetos, pois permitem que o aluno vivencie na prática os conceitos sendo este o personagem principal do ensino-aprendizado, tendo o professor como um auxiliador do processo. Nesse contexto, o aluno consegue aprender no seu tempo e de acordo com o seu interesse no assunto, sendo instigado a pesquisar por conta própria até descobrir a solução para o determinado contexto.

Como contribuições para o trabalho, a partir das experimentações realizadas em sala de aula, percebeu-se também que há uma demanda maior de tempo para que o profes- 
VIII Congresso Brasileiro de Informática na Educação (CBIE 2019)

Anais do XXV Workshop de Informática na Escola (WIE 2019)

sor prepare aulas dinâmicas e que saiba utilizar materiais diferenciados para a produção e a aplicação das metodologias híbridas. Dessa forma, espera-se continuar a pesquisa em busca de objetos de aprendizagem que auxiliem o professor nesses contextos.

\section{Referências}

Abdullah, A. and Yih, T. Y. (2014). Implementing learning contracts in a computer science course as a tool to develop and sustain student motivation to learn. Procedia Social and Behavioral Sciences, 123:256-265.

Bacich, L. and Moran, J. (2018). Metodologias Ativas para uma Educação Inovadora: Uma Abordagem Teórico-Prática. Penso.

Benlloch-Dualde, J., Buendía, F., and Cano, J. (2010). On the design of interactive classroom environments based on the tablet pc technology. In 2010 IEEE Frontiers in Education Conference (FIE), pages T4C-1-T4C-6.

Chen, G. and Weng, W. (2008). Reform and practice in teaching j2ee programming. In 2008 The 9th International Conference for Young Computer Scientists, pages 25072511.

Coldwell, J., Craig, A., and Goold, A. (2011). Using e technologies for active learning. Interdisciplinary journal of information, knowledge and management, 6:95-106.

de Oliveira Pinto, D. (2017). Entenda a importância e o papel das metodologias ativas de aprendizagem.

de Oliveira Pinto, D. (2019). O que é e como aplicar o ensino híbrido?

Drini, M. (2018). Using new methodologies in teaching computer programming. In 2018 IEEE Integrated STEM Education Conference (ISEC), pages 120-124.

Gouvea, E. P., Odagim, A. M., Shitsuka, D. M., and Shitsuka, R. (2015). Um trabalho de pesquisa ação com uso de metodologia ativa no ensino de tecnologia da informação. Educação, Gestão e Sociedade: revista da Faculdade Eça de Queirós, 5(20):13p.

Gudigantala, N. (2013). An active learning approach to teaching undergraduate introduction to mis course. In Proceedings of the Nineteenth Americas Conference on Information Systems, pages 1-9.

Jonsson, H. (2015). Using flipped classroom, peer discussion, and just-in-time teaching to increase learning in a programming course. In 2015 IEEE Frontiers in Education Conference (FIE). IEEE.

Krugel, J. and Hubwieser, P. (2017). Computational thinking as springboard for learning object-oriented programming in an interactive MOOC. In 2017 IEEE Global Engineering Education Conference (EDUCON). IEEE.

Mok, H. N. (2014). Teaching tip: The flipped classroom. Journal of Information Systems Education, 25(1):7-11.

Santos, N. S., Ferreira, L. S., and Prates, R. O. (2012). An overview of evaluation methods for collaborative systems. In 2012 Brazilian Symposium on Collaborative Systems, pages 127-135.

Virginia, T. (2016). O que é metodologia ativa e por que ela é tão importante em uma graduação. 